\section{Low temperature physics}

The Quest for Absolute Zero: The Meaning of Low Temperature Physics. Second edition with SI Units. By K. Mendelssohn. Pp. 281. (Taylor and Francis: London, 1977.) $£ 5$

Low temperature physics, for those who know little of it, often seems to represent the very epitome of scientific futility: a seemingly purposeless (and highly expensive) competition to see who can approaich most closely the unattainable absolute zero of temperature. They should read this book. It would be very likely to change their minds since, as an apologia for one of the most fascinating and fruitful areas of physics to emerge this century, it could hardly be bettered.

Mendelssohn sets low temperature physics squarely in its historical and scientific context, close to the centre of the stage during that quite unprecedented explosion of knowledge which took place in physics in the early decades of the present century. Using an entirely non-mathematical approach, he explains with impressive clarity and accuracy how many of the fundamental concepts of modern physics arose from, or were illuminated and supported by, experiments on the novel states of matter which exist at very low temperatures.

The first four chapters are largely historical in structure, the story opening with Cailletet's liquefaction of oxygen $(90 \mathrm{~K})$ in 1877 , running on to describe the liquefaction of hydrogen $(20 \mathrm{~K})$ by Dewar in 1898 . This first section ends with an assessment of the achievements of Kamerlingh Onnes who, by tiquefying helium (4K) in 1908 and discovering superconductivity, became the father of low temperature physics as we know it today. This section includes explanations of the isotherms of real (non-ideal) gases, critical points, cascade liquefiers and expansion engines.

Unusually, Mendelssohn considers how the personal attributes of the researchers may crucially have influenced the extent of their scientific achievements. For example, Dewar, portrayed as a brilliant but autocratic and quarrelsome loner, failed in his attempt to liquefy helium. The prize fell, instead, to the much more open, equable and diplomatic Onnes who possessed the inclination and ability to create a scientific laboratory in the modern sense, suocessfully coordinating the activities of a comparatively large complement of technical staff, colleagues, and visitors from other universities.

The next five chapters include vigorous and readily understandable accounts of topics such as the Third Law, heat, entropy, zero point energy, low temperature specific heats, quantisation, radiation, indeterminacy, gas degeneracy, quantum statistics of ideal gases, Fermi surfaces, Curie's Law, magnetic cooling and the various ramifications of superconduotivity. Individual contributions to the developing coniceptual structure made by such figures as Bohr, Born, de Broglie, Einstein, Fermi, Heisenberg, Nernst and Schroedinger, many of whom were known personally to the author, are carefully described and assessed.

At this point, the second edition diverges from the original, published in 1966. A whole chapter is now devoted to cryogenic technology, dealing with SQUIDs (superconducting quantum interference devices, used for measuring tiny magnetic fields), computer elements, superconducting motors and generators and, of course, superconducting magnets. The final chapter, on superfuidity, has also been extensively reworked to take account of recent advances, panticularly in the new and rapidly growing field of superfluid helium-3. Even so, the pace of current cryogenic development seems

\section{Mammalian development}

Developments in Mammals. Vol. 2. Edited by Martin H. Johnson. Pp. 241. (North-Holland: Amsterdam, New York and Oxford, 1977.) Dfl. 72; $\$ 29.50$.

THE second volume in this interesting and timely series continues in the style and format established in the first. There are both good and bad aspects to this. It was hoped that the small and inadequate typeface would have been improved but this volume is also difficult and tiring to read. Such is the speed with which these two volumes have been produced that the changes in typeface could not be introduced in volume 2 . I for one look forward to their introduction in the next issue.

The good balanced mixture of developmental biology, reproductive physiology and biochemistry has been maintained. Two papers concern growth and maturation of oocytes, and transport and selection of sperm. These are followed by three papers each considering the cleavage stages of rodent development from slightly different viewpoints. This section is particularly welcome as the subject is somewhat controversial, with much circumstantial evidence concerning determination and differentiation of the two cell lines which emerge during cleavage. Here, in these three papers, all the to have outstripped production of the buok which, for instance, describes dilution refrigeration as capable of "temperatures approaching $0.01 \mathrm{~K}$ in continuous operation", whereas $0.003 \mathrm{~K}$ is now routinely being reached by this technique at Grenoble.

The colouned line drawings are helpful. There are also a number of photographs portraying most of the major scientific figures discussed in the text: of particular note is one entitled: "The heyday of low temperature physics. L. D. Landau in discussion with the author in Moscow, 1957."

As might be expected in a work with this breadith of vision, containing so much of the author's personal viewpoint of the scientific world, pungently and entertainingly expressed, there is much with which practitioners may take issue; but this in no way detracts from the success of the book as a whole. Like the previous edition, it can confidently be recommended to anyone who would like to know more about the behaviour of matter in that unimaginable abyss of coldness around and below $1 \mathrm{~K}$.

P. V. E. McClintock

P. V. E. McClintock is a member of the Department of Physics at the University of Lancaster, $\boldsymbol{U K}$. evidence is reviewed, and the various interpretations that have been put on the data are well discussed. Of the remaining four papers, one reviews the in vitro culture systems and their application to the peri-implantation period, whereas the other three address another hotly controversial subject - the involvement of steroid hormones in controlling implantation and in particular to what extent the mammalian blastocyst may produce steroids and thus perhaps control, at least in part, its own implantation.

Encouraged by editorial policy, authors have occasionally been speculative and have also used the book as an outlet for previously unpublished data. Although this policy has obvious advantages in publications such as this series, there are hazards since papers are edited rather than refereed, and somewhat one-sided arguments occasionally slip through the net. In this second volume, the editor has provided the best possible answer to thsi problem by gathering together several papers on one subject, thus presenting argument and counter-argument side by side. For scientists new to the field, this is especially useful. Although expensive, this volume is good value and augurs well for the next of the series.

Michael H. L. Snow

Michael Snow is a Research Scientist with the MRC Mammalian Development Unit, London, UK. 